eISSN 2236-5257

10.46551/ruc.v22n1a03

\title{
Fatores associados à necessidade de hemotransfusão em recém-nascidos cadastrados em um ambulatório de follow-up
}

\section{Factors associated with need for blood transfusion in newborns registered at a follow-up clinic}

\author{
Antônio Prates Caldeira ${ }^{1}$ \\ Andreia Caroline Ribeiro Ramos ${ }^{2}$ \\ Isabella Prates Caldeira ${ }^{3}$ \\ Patrícia Soares Castro ${ }^{4}$ \\ Jair Almeida Carneiro ${ }^{5}$ \\ Lucineia de Pinho ${ }^{6}$
}

Resumo: Objetivo: conhecer a frequência e os fatores associados à necessidade de hemotransfusão em recém-nascidos acompanhados em um serviço seguimento de recémnascidos de alto risco. Método: trata-se de estudo com base em dados secundários, a partir do grupo de crianças de ambulatório de seguimento no norte de Minas Gerais. Foram coletadas variáveis relacionadas às condições de gestação, parto e permanência na Unidade de Terapia Intensiva Neonatal. Após análises bivariadas, seguiu-se regressão de Poisson com as variáveis que se mostraram associadas até o nível de $20 \%$. No modelo final, permaneceram apenas as variáveis que se mostraram associadas à necessidade de hemotransfusão até o nível de 5\%, registrando-se as Razões de Prevalência (RP) e seus respectivos intervalos de confiança de 95\%. Resultados: foram coletados dados de 282 neonatos. Houve ligeiro predomínio do sexo

\footnotetext{
${ }^{1}$ Doutor em Pediatria (UFMG). Professor da Universidade Estadual de Montes Claros (Unimontes). Minas Gerais. Brasil. $\square$ antonio.caldeira@ unimontes.br. (i) https://orcid.org/0000-0002-9990-9083.

${ }^{2}$ Graduanda em Medicina (Unimontes). Bolsista de Iniciação Científica da Universidade Estadual de Montes Claros (Unimontes). Minas Gerais. Brasil. $\square$ andreia897@hotmail.com. (D) https://orcid.org/0000-0001-78502340.

${ }^{3}$ Graduanda em Medicina (UNIFIPMOC). Bolsista de Iniciação Científica do Centro Universitário FIPMoc (UNIFIPMOC). Minas Gerais. Brasil. $₫$ isabellapratescaldeira@gmail.com (D) https://orcid.org/0000-00020421-6728.

${ }^{4}$ Mestre em Cuidado Primário em Saúde (Unimontes) Professor do Centro Universitário FIPMoc (UNIFIPMOC). Minas Gerais. Brasil. $₫$ patcastro83@ hotmail.com (iD https://orcid.org/0000-0002-5364-4737. ${ }^{5}$ Doutor em Ciências da Saúde (Unimontes). Universidade Estadual de Montes Claros (Unimontes). Minas Gerais. Brasil. $₫$ jairjota@yahoo.com.br (D) https://orcid.org/0000-0002-9501-918X.

${ }^{6}$ Doutor em Ciências da Saúde (Unimontes). Universidade Estadual de Montes Claros (Unimontes). Minas Gerais. Brasil. $₫ \underline{\text { lucineiapinho@ hotmail.com (D) https://orcid.org/0000-0002-2947-5806 }}$

\begin{tabular}{cccc}
\hline Recebido em & Correções em & Aceito em & Publicado em \\
$27 / 05 / 2020$ & $09 / 06 / 2020$ & $11 / 06 / 2020$ & $16 / 06 / 2020$
\end{tabular}
}


masculino. Mais da metade apresentavam peso de nascimento abaixo de 1500 gramas $(59,6 \%)$. Entre as intercorrências apresentadas, a sepse foi a mais comum $(58,9 \%)$. A anemia com necessidade de hemotransfusão foi identificada em 96 prontuários (34,0\%). Em uma análise conjunta, as variáveis que se mostraram estatisticamente associadas à necessidade de hemotransfusão para o grupo estudado foram: o peso de nascimento menor que $1500 \mathrm{~g}$ $(\mathrm{RP}=1,25$; IC95\%:1,09-1,39), o tempo de oxigenioterapia igual ou superior a 15 dias $(\mathrm{RP}=1,42$; IC95\%:1,29-1,56) e a ocorrência de sepse (RP=1,11; IC95\%:1,01-1,23). Conclusão: observouse elevada frequência de hemotransfusão para o grupo estudado. As variáveis associadas destacam o papel da prematuridade e dos cuidados com os prematuros no período neonatal.

Palavras-chave: Transfusão de sangue; Recém-nascido prematuro; Anemia; Terapia intensiva neonatal.

Abstract: Objective: to know the frequency and factors associated with the need for blood transfusion in newborns monitored in a high-risk newborn follow-up service. Method: this is a study based on secondary data, from the group of children in an outpatient follow-up clinic in northern Minas Gerais. Variables related to the conditions of pregnancy, delivery and stay in the Neonatal Intensive Care Unit were collected. After bivariate analyzes, Poisson regression was followed with the variables that were associated up to the level of $20 \%$. In the final model, only the variables that were associated with the need for blood transfusion up to the level of 5\% remained, registering the Prevalence Ratios (PR) and their respective 95\% confidence intervals. Results: data were collected from 282 neonates. There was a slight predominance of males. More than half had birth weight below 1500 grams (59.6\%). Among the complications presented, sepsis was the most common (58.9\%). Anemia requiring blood transfusion was identified in 96 medical records (34.0\%). In a joint analysis, the variables that were shown to be statistically associated with the need for blood transfusion for the studied group were: birth weight less than $1500 \mathrm{~g}(\mathrm{PR}=1.25 ; 95 \% \mathrm{CI}$ : 1.09-1.39), time of oxygen therapy equal to or greater than 15 days ( $\mathrm{PR}=1.42 ; 95 \% \mathrm{CI}$ : 1.29-1.56) and the occurrence of sepsis $(\mathrm{PR}=1.11$; 95\% CI: 1.01-1.23). Conclusion: there was a high frequency of blood transfusion for the studied group. The associated variables highlight the role of prematurity and care for premature infants in the neonatal period.

Key word: Blood Transfusion; Infant, Premature; Anemia; Intensive Care, Neonatal 


\section{INTRODUÇÃO}

Nos últimos anos, tem sido cada vez mais frequente a sobrevida de recém-nascidos muito prematuros e de muito baixo peso, graças aos avanços tecnológicos e medidas assumidas pelas Unidades de Terapia Intensiva Neonatal (UTIN) ${ }^{1}$. Entretanto, nessas unidades, os recémnascidos apresentam muitas intercorrências e necessitam de intervenções complexas e que podem culminar em impactos negativos ao longo da vida ${ }^{2-4}$.

Uma condição muito comumente observada em recém-nascidos prematuros é a anemia, com necessidade de transfusão de concentrados de hemácias. Todavia, eventualmente, as hemotransfusões em recém-nascidos prematuros se associam a efeitos adversos e até ao óbito ${ }^{5,6}$. Não se trata, portanto, de uma medida orientada rotineiramente e seu uso racional criterioso deve ser a meta para todos os profissionais que atuam em $\mathrm{UTIN}^{7}$.

Considerando os riscos inerentes à hemotransfusões para recém-nascidos prematuros e de baixo peso, é importante conhecer os fatores associados à necessidade desse procedimento. Um estudo realizado em hospitais universitários, no Brasil, revelou que cerca de metade das crianças acompanhadas em UTIN receberam transfusões de hemácias e que os fatores associados à necessidade da intervenção foram a idade gestacional, o escore de gravidade dos prematuros, o registro de apneia, hemorragia pulmonar ou intraventricular, sepse, necessidade de oxigênio aos 28 dias de vida, enterocolite necrosante, ventilação mecânica, uso de cateter umbilical, nutrição parenteral, a unidade onde está internada a criança e a duração da internação superior a $60 \operatorname{dias}^{8}$.

Apesar de todos os fatores associados, os autores concluem, destacando a grande variabilidade entre as instituições envolvidas no estudo, registrando, também, que mesmo depois de ajustar para condições adversas de saúde e intervenções terapêuticas, a unidade neonatal continuou a influenciar as práticas de hemotransfusão.

A região norte de Minas Gerais possui algumas UTIN, mas, ainda, não conta com nenhum estudo que avalie aspectos correlatos à anemia e hemotransfusão em neonatos. Os resultados de um estudo de tal natureza têm o potencial de auxiliar e nortear as condutas dos profissionais de saúde. A cidade polo da região norte de Minas Gerais conta com o único centro de acompanhamento de recém-nascidos egressos de UTIN. No presente estudo, objetivou-se 
conhecer a frequência e os fatores associados à necessidade de hemotransfusão em recémnascidos acompanhados em um serviço seguimento de recém-nascidos de alto risco.

\section{MÉTODO}

Trata-se de uma pesquisa exploratória conduzida a partir do acompanhamento de recémnascidos assistidos pelo ambulatório de seguimento de recém-nascidos de alto risco. Neste serviço, são acompanhadas crianças egressas das UTIN da cidade, com assistência interdisciplinar. Os dados foram obtidos através da análise dos prontuários das crianças cadastradas a partir da alta da UTIN, entrevistas com as mães, e, eventualmente, consultas aos prontuários hospitalares (quando faltavam dados nos prontuários ambulatoriais e as mães não sabiam informar).

Não houve cálculo amostral e a seleção de prontuários alcançou todos os recémnascidos prematuros egressos das UTIN da cidade nos cinco anos antecedentes à coleta de dados (2014 a 2018), com seguimento ao longo do primeiro ano de vida. Foram excluídas as crianças cujas mães não tinham informações precisas sobre as condições de gestação e nascimento e não apresentavam os dados faltantes registrados nos respectivos prontuários. A limitação temporal teve o objetivo de amenizar o viés de memória, excluindo-se crianças de idades mais avançadas, com registro de nascimento anterior ao ano de 2014.

O instrumento de coleta de dados foi construído a partir dos objetivos específicos do estudo, abordando características maternas e condições de gestação (paridade materna, intercorrências durante a gestação, uso de corticoide pré-natal), parto (tempo de bolsa rota, via de parto), características do recém-nascido (sexo, idade gestacional, peso de nascimento, Apgar no quinto minuto) e intercorrências no pós-parto, durante a permanência nas UTIN (necessidade de ventilação mecânica, tempo de oxigenioterapia, uso de aminas e registro de sepse). A variável resposta foi o registro de hemotransfusão, durante o período de estadia na UTIN.

A coleta de dados foi realizada por dois estudantes de iniciação científica, especialmente treinados para identificação dos dados em prontuários e entrevistas com as mães, e, eventualmente, esclarecimentos junto à equipe de profissionais que acompanhavam as crianças. O período de coleta de dados foi de fevereiro a dezembro de 2018. 
Após análises bivariadas, seguiu-se análise de regressão múltipla de Poisson, com variância robusta, considerando todas as variáveis que se mostraram associadas até o nível de $20 \%$ na etapa inicial. Para o modelo final, o nível de significância foi definido em 5\%. Todos os dados coletados foram codificados e analisados através do software IBM-SPSS for Windows versão 22.0 .

Todas as considerações éticas foram levadas em consideração, dentro dos padrões exigidos pela declaração de Helsinki e o projeto da pesquisa foi apreciado e aprovado pelo Comitê de Ética em Pesquisa da instituição sede do estudo, a Universidades Estadual de Montes Claros - Unimontes, conforme o parecer $\mathrm{n}^{\mathrm{o}} 1.800 .915$.

\section{RESULTADOS}

Foram avaliados dados de 282 crianças nascidas prematuramente. Na análise dos dados das gestantes, registrou-se que a maior parte delas já tinham registro de parto anterior $(58,2 \%)$. Mais da metade das gestantes $(57,1 \%)$ informaram alguma intercorrência durante a gestação, sendo a hipertensão arterial a mais comum delas $(35,8 \%)$. O tipo de parto foi predominantemente por via operatória $(67,7 \%)$ (TAB. 1) 
Tabela 1: Características maternas e condições de gestação e parto para crianças acompanhadas em Ambulatório de Follow-up - Montes Claros (MG), 2014-2018.

\begin{tabular}{|c|c|c|}
\hline Variáveis & (n) & $(\%)$ \\
\hline \multicolumn{3}{|l|}{ Gestações prévias } \\
\hline Nenhuma & 118 & 41,8 \\
\hline Uma ou mais & 164 & 58,2 \\
\hline \multicolumn{3}{|c|}{ Intercorrências maternas na gestação } \\
\hline Hipertensão & 101 & 35,8 \\
\hline Diabetes & 13 & 4,6 \\
\hline Infecção & 25 & 8,9 \\
\hline Nenhuma referida & 121 & 42,9 \\
\hline Outros & 22 & 7,8 \\
\hline \multicolumn{3}{|c|}{ Uso de corticoide pré-natal } \\
\hline Sim & 142 & 50,4 \\
\hline Não & 140 & 49,6 \\
\hline \multicolumn{3}{|l|}{ Tempo de bolsa rota } \\
\hline$<18$ horas & 215 & 76,2 \\
\hline$\geq 18$ horas & 67 & 23,8 \\
\hline \multicolumn{3}{|l|}{ Tipo de parto } \\
\hline Normal & 91 & 32,3 \\
\hline Cesárea & 191 & 67,7 \\
\hline \multicolumn{3}{|c|}{ Idade gestacional (semanas) } \\
\hline $25-28$ & 71 & 25,2 \\
\hline 29-32 & 129 & 45,7 \\
\hline $33-36$ & 73 & 25,9 \\
\hline$\geq 37$ & 9 & 3,2 \\
\hline
\end{tabular}

Os dados das crianças avaliadas registram ligeiro predomínio do sexo masculino $(53,9 \%)$. Quase metade dos recém-nascidos necessitaram medidas de reanimação, ainda em sala de parto (47,9\%). Mais da metade apresentavam peso de nascimento abaixo de 1500 gramas $(59,6 \%)$. Entre as intercorrências apresentadas na UTIN, a sepse foi a mais comum (58,9\%). A anemia com necessidade de hemotransfusão foi identificada em 96 prontuários $(34,0 \%)$ (TAB. 2$)$. 
Tabela 2: Caracterização e intercorrências neonatais de crianças acompanhadas em Ambulatório de Follow-up - Montes Claros (MG), 2014-2018.

\begin{tabular}{|c|c|c|}
\hline Variáveis & (n) & $(\%)$ \\
\hline \multicolumn{3}{|l|}{ Sexo } \\
\hline Masculino & 152 & 53,9 \\
\hline Feminino & 130 & 46,1 \\
\hline \multicolumn{3}{|l|}{ Apgar $5^{\circ} \mathrm{min}$} \\
\hline$\geq 7$ & 196 & 69,5 \\
\hline$<7$ & 86 & 30,5 \\
\hline \multicolumn{3}{|c|}{ Necessidade de reanimação em sala de parto } \\
\hline Não & 147 & 52,1 \\
\hline Sim & 135 & 47,9 \\
\hline \multicolumn{3}{|l|}{ Peso ao nascer } \\
\hline$<1000 \mathrm{~g}$ & 59 & 20,9 \\
\hline $1000-1499 \mathrm{~g}$ & 109 & 38,7 \\
\hline $1500-2499 \mathrm{~g}$ & 101 & 35,8 \\
\hline$>2500 \mathrm{~g}$ & 13 & 4,6 \\
\hline \multicolumn{3}{|c|}{ Classificação RN } \\
\hline AIG & 187 & 66,3 \\
\hline PIG & 85 & 30,1 \\
\hline GIG & 10 & 3,5 \\
\hline \multicolumn{3}{|c|}{ Tempo de oxigenioterapia na UTIN } \\
\hline$<7$ dias & 101 & 35,8 \\
\hline $7-14$ dias & 46 & 16,3 \\
\hline 15-29 dias & 71 & 25,2 \\
\hline$\geq 30$ dias & 64 & 22,7 \\
\hline \multicolumn{3}{|c|}{ Uso de aminas vasoativas } \\
\hline Não & 191 & 67,7 \\
\hline Sim & 91 & 32,3 \\
\hline \multicolumn{3}{|c|}{ Sepse com uso de antimicrobianos } \\
\hline Não & 116 & 41,1 \\
\hline Sim & 166 & 58,9 \\
\hline \multicolumn{3}{|c|}{ Anemia com registro de hemotransfusão } \\
\hline Não & 186 & 66,0 \\
\hline Sim & 96 & 34,0 \\
\hline
\end{tabular}


As variáveis que, por meio de análises bivariadas, se mostraram associadas a necessidade de hemotransfusão até o nível de 20\% (TAB 3) foram selecionadas para avaliação, de forma conjunta, por meio da regressão de Poisson, com variância robusta, admitindo-se ao final o nível de significância de 5\%.

Tabela 3 - Associação entre características do grupo estudado e necessidade de hemotransfusão em prematuros acompanhados em Ambulatório de Follow-up (Análise bivariada) - Montes Claros (MG), 2014-2018.

\begin{tabular}{|c|c|c|c|c|c|c|}
\hline \multirow[t]{3}{*}{ Variável } & \multicolumn{4}{|c|}{ Necessidade de hemotransfusão } & \multirow[t]{3}{*}{$\mathrm{p}$ valor } & \multirow[t]{3}{*}{ RP (IC 95\%) Bruta } \\
\hline & \multicolumn{2}{|c|}{ Sim } & \multicolumn{2}{|c|}{ Não } & & \\
\hline & (n) & $(\%)$ & (n) & $(\%)$ & & \\
\hline Sexo & & & & & 0,949 & \\
\hline Feminino & 44 & 33,8 & 86 & 66,2 & & $0,99(0,71-1,37)$ \\
\hline Masculino & 52 & 34,2 & 100 & 65,8 & & 1,0 \\
\hline Paridade materna & & & & & 0,082 & \\
\hline Primípara & 47 & 39,8 & 71 & 60,2 & & $1,33(0,97-1,84)$ \\
\hline Multípara & 49 & 29,9 & 115 & 70,1 & & 1,0 \\
\hline Tempo de bolsa rota (horas) & & & & & 0,016 & \\
\hline$>18$ & 31 & 46,3 & 36 & 53,7 & & $1,53(1,10-2,13)$ \\
\hline$\leq 18$ & 65 & 30,2 & 150 & 69,8 & & 1,0 \\
\hline Tipo de parto & & & & & 0,587 & \\
\hline Operatório & 63 & 33,0 & 128 & 67,0 & & $0,91(0,65-1,28)$ \\
\hline Normal & 33 & 36,3 & 58 & 63,7 & & 1,0 \\
\hline Hipertensão na gestação & & & & & 0,375 & \\
\hline Sim & 31 & 30,7 & 70 & 69,3 & & $0,86(0,60-1,22)$ \\
\hline Não & 65 & 35,9 & 116 & 64,1 & & 1,0 \\
\hline Reanimação ao nascer & & & & & 0,043 & \\
\hline Sim & 54 & 40,0 & 81 & 60,0 & & $1,40(1,01-1,95)$ \\
\hline Não & 42 & 28,6 & 105 & 71,4 & & 1,0 \\
\hline Tempo de oxigenioterapia (dias & & & & & $<0,001$ & \\
\hline$\geq 15$ & 71 & 52,6 & 64 & 47,4 & & $3,09(2,09-4,57)$ \\
\hline$<15$ & 25 & 17,0 & 122 & 83,0 & & 1,0 \\
\hline Uso de Ventilação mecânica & & & & & $<0,001$ & \\
\hline Sim & 72 & 46,5 & 83 & 53,5 & & $2,46(1,65-3,66)$ \\
\hline Não & 24 & 18,9 & 103 & 81,1 & & 1,0 \\
\hline Peso de nascimento (gramas) & & & & & $<0,001$ & \\
\hline
\end{tabular}




\begin{tabular}{|c|c|c|c|c|c|c|}
\hline$<1500$ & 79 & 47,0 & 89 & 53,0 & & $3,15(1,95-5,03)$ \\
\hline$\geq 1500$ & 17 & 14,9 & 97 & 85,1 & & 1,0 \\
\hline Idade gestacional (semanas) & & & & & $<0,001$ & \\
\hline$\leq 32$ & 83 & 41,5 & 117 & 58,5 & & $2,62(1,55-4,43)$ \\
\hline$>32$ & 13 & 15,9 & 69 & 84,1 & & 1,0 \\
\hline Uso de aminas & & & & & $<0,001$ & \\
\hline Sim & 44 & 48,4 & 47 & 51,6 & & $1,78(1,30-2,43)$ \\
\hline Não & 52 & 27,2 & 139 & 72,8 & & 1,0 \\
\hline Longa permanência & & & & & 0,001 & \\
\hline Sim & 15 & 65,2 & 8 & 34,8 & & $2,09(0,47-2,96)$ \\
\hline Não & 81 & 31,3 & 178 & 68,7 & & \\
\hline Sepse & & & & & $<0,001$ & \\
\hline Sim & 71 & 42,8 & 95 & 57,2 & & $1,96(1,35-2,93)$ \\
\hline Não & 25 & 21,6 & 91 & 78,4 & & 1,0 \\
\hline
\end{tabular}

Os resultados das análises múltiplas revelaram que, em uma análise conjunta, as variáveis que se mostraram estatisticamente associadas à necessidade de hemotransfusão para o grupo estudado foram: o peso de nascimento menor que 1500g ( $\mathrm{RP}=1,25$; IC95\%:1,09-1,39), o tempo de oxigenioterapia igual ou superior a 15 dias ( $\mathrm{RP}=1,42$; IC95\%:1,29-1,56) e a ocorrência de sepse na UTIN (RP=1,11; IC95\%:1,01-1,23) (TAB 4). 
Tabela 4 - Associação entre características do grupo estudado e necessidade de hemotransfusão em prematuros acompanhados em Ambulatório de Follow-up (Análise de Regressão de Poisson) - Montes Claros (MG), 2014-2018.

\begin{tabular}{lcc}
\hline Variável & p-valor & RP (IC 95\%) ajustada \\
\hline Peso & 0,001 & \\
$\quad<1500 \mathrm{~g}$ & & $1,25(1,09-1,39)$ \\
$\quad \geq 1500 \mathrm{~g}$ & $<0,001$ & 1,0 \\
Tempo de oxigenioterapia & & $1,42(1,29-1,56)$ \\
$\quad \geq 15$ dias & & 1,0 \\
$\quad<15$ dias & 0,048 & \\
Sepse & & $1,11(1,01-1,23)$ \\
Sim & & 1,0 \\
Não & &
\end{tabular}

\section{DISCUSSÃO}

Este estudo identificou uma elevada frequência de anemia com necessidade de hemotransfusão entre as crianças acompanhadas pelo ambulatório de seguimento de recémnascidos de alto risco. Todavia, outros estudos mostram percentuais até mais elevados do que foi observado no presente estudo. Em estudo que abordou o mesmo tema em hospitais universitários no Brasil, a frequência de hemotransfusões em recém-nascidos de muito baixo peso variou de $34,1 \%$ a $66,4 \%{ }^{8}$. Em outro estudo realizado na Espanha, também com recémnascidos de muito baixo peso, a frequência observada foi de $53,6 \%{ }^{9}$. Por outro lado, existem estudos com frequências mais baixas, como $39,4 \%{ }^{10}, 21,1 \%^{11}$ ou $20,9 \%^{12}$.

Frequências mais elevadas de hemotransfusões são registradas em recém-nascidos prematuros e de muito baixo peso. Desde o momento do nascimento, essas crianças apresentam maiores riscos para a anemia, a partir de uma ligadura muito precoce do cordão umbilical ${ }^{13}$, passando pelas intervenções com coletas de sangue, intercorrências infecciosas e a própria imaturidade da medula, com reduzida capacidade de produção de hemácias ${ }^{14}$.

Apesar dessas considerações, as indicações para hemotransfusões devem ser restritas segundo as mais recentes orientações ${ }^{15}$. Um estudo de revisão sistemática da literatura com metanálise concluiu que estratégias mais liberais para uso de hemoderivados em prematuros 
não se mostrou superior à prática restritiva de transfusão em estudos randomizados para taxas de retinopatia de prematuridade, doença pulmonar crônica ou hemorragia intraventricular ${ }^{16}$. Um estudo nacional concluiu que a necessidade de transfusões de hemácias em prematuros de muito baixo peso, ao nascer, esteve associada tanto às condições clínicas do neonato como às próprias unidades de saúde, e, os autores advogam que o número de transfusões durante a internação pode ser usado como uma medida da qualidade dos cuidados neonatais ${ }^{17}$.

Em relação aos fatores associados à necessidade de hemotransfusão entre as crianças do presente estudo, o peso de nascimento abaixo de 1500 gramas pode ser considerado um marcador direto de maior prematuridade entre as crianças avaliadas. Alguns estudos limitam a investigação sobre a necessidade de hemotransfusão exclusivamente a esse grupo de neonatos $5,9,17,18$. É razoável assumir que quanto maior o grau de prematuridade e/ou mais baixo peso, maiores são as chances para que os prematuros sejam submetidos a uma hemotransfusão. Além da velocidade de crescimento somático desse grupo de crianças, com consequente aumento do volume sanguíneo e reduzido tempo de vida de suas células sanguíneas, deve-se considerar ainda as espoliações frequentes para exames e os níveis baixos de eritropoetina ${ }^{14}$.

O uso prolongado de oxigenioterapia, também, tem sido apontado em outros estudos, embora com outros pontos de corte ${ }^{5,17}$. O suporte ventilatório representa um aspecto crucial para a sobrevida de grande parte dos recém-nascidos prematuros. Sua associação à indicação da hemotransfusão é pertinente, conquanto o principal objetivo desta é aumentar a liberação de oxigênio em nível tecidual ${ }^{18}$. Deve-se considerar ainda que o uso prolongado de oxigenioterapia, quase sempre por meio de ventilação mecânica, se associa a maiores riscos de traumas e intercorrências hemorrágicas e infecciosas.

Em relação à associação da necessidade de hemotransfusão com sepse, também é um achado já apontado pela literatura ${ }^{10,12}$. Para os neonatos criticamente enfermos e/ou quadros infecciosos graves, a liberação de algumas citocinas tendem a inibir a eritropoise, que já é restrita $^{19,20}$. É relevante destacar, entretanto, que alguns autores questionam tal associação, sugerindo que a indicação estaria muito mais associada às condições clínicas globais do neonato do que ao quadro de sepse clássica ${ }^{12}$. Nesse sentido, é relevante destacar a necessidade de diagnóstico acurado para a sepse e, igualmente, a necessidade dos cuidados para evitá-la.

Outros estudos mostraram, ainda, associação com outras variáveis, como por exemplo, idade gestacional, gravidade dos prematuros, apneia, hemorragia pulmonar ou intraventricular, enterocolite necrosante, uso de cateter umbilical, nutrição parenteral ${ }^{5,17}$. Mas são restritos 
apenas a recém-nascidos de muito baixo peso. Outros autores observaram que não houve associações estatisticamente significativas entre transfusões e ganho de peso neonatal, apneia e suporte respiratório ${ }^{11}$. Esses achados diversos devem ser avaliados no contexto de cada instituição, que deve buscar compreender as implicações de cada um dos fatores associados, como medida de qualidade do cuidado, conforme apontado por Santos et $\mathrm{al}^{17}$.

É imperioso, portanto, que as UTIN busquem construir protocolos de monitoramento de suas práticas e de vigilância ao uso excessivo de hemoderivados, considerando que as recomendações recentes são mais restritivas ${ }^{15,16,19}$. A adoção de protocolos mais restritivos para transfusões de hemácias constitui uma medida útil para reduzir o número de transfusões em neonatos, mas não deve ser assumida como medida isolada ${ }^{21,22}$.

Os resultados do presente estudo devem ser considerados à luz de algumas limitações. Trata-se de um estudo restrito ao grupo de neonatos sobreviventes, que fazem parte do grupo acompanhado em ambulatório de seguimento. Infelizmente, alguns prematuros têm um óbito precoce ou não são acompanhados pelo serviço local. Trata-se, também, de um estudo fundamentado em dados secundários, que não foram previamente definidos para pesquisas. Apesar dessas limitações, os resultados são relevantes e devem ser utilizados por gestores e profissionais de saúde para melhoria contínua da prática e dos cuidados neonatais.

\section{CONCLUSÃO}

Identificou-se que uma elevada proporção de recém-nascidos, acompanhados pelo ambulatório de seguimento, apresentaram necessidade de hemotransfusão, durante a estadia na UTIN. Os fatores associados à hemotransfusão foram o peso de nascimento muito baixo, o tempo de oxigenioterapia igual ou superior a 15 dias e ocorrência de sepse. Esses fatores destacam o papel da prematuridade como principal fator de risco para a hemotransfusão e a necessidade de melhoria dos cuidados com os prematuros no período neonatal.

\section{FINANCIAMENTO}

Fundação de Amparo à Pesquisa do Estado de Minas Gerias - FAPEMIG. Processo nº: APQ:02030/15. 


\section{CONFLITO DE INTERESSES}

Os autores declaram que não existem conflitos de interesses.

\section{REFERÊNCIAS}

1 HORBAR, Jeffrey D.; et al. Mortality and neonatal morbidity among infants 501 to 1500 grams from 2000 to 2009. Pediatrics, v. 129, n. 6, p. 1019-1026, 2012.

2 KEIR, Amy; MCPHEE, Andrew; WILKINSON, Dominic. Beyond the borderline:

Outcomes for inborn infants born at $\leq 500$ grams. J Paediatr Child Health, v. 50, n. 2, p. 146-152, 2014.

3 DOYLE, Lex W.; et al. Neonatal intensive care at borderline viability - is it worth it? Early Hum Dev, v. 80, n. 2, p. 103-113, 2004.

4 KHAN, Rizwan A.; et al. Resuscitation at the limits of viability - an Irish perspective. Acta Paediatr, v. 98, n. 9, p. 1456-1460, 2009.

5 SANTOS, Amélia M.; et al. Red blood cell transfusions are independently associated with intra-hospital mortality in very low birth weight preterm infants. J Pediatr, v. 159, n. 3, p. 371-376, 2011.

6 MOHAMED, Adel; SHAH, Parkesh S. Transfusion associated necrotizing enterocolitis: a meta-analysis of observational data. Pediatrics, v. 129, n. 3, p. 529-40, 2012.

7 AUCOTT, Susan W.; MAHESHWARI, Akhil. To transfuse or not transfuse a premature infant: the new complex question. J Perinatol, v. 39, n. 3, p. 351-353, 2019.

8 SANTOS, Amélia Miyashiro N.; et al. Factors associated with red blood cell transfusions in very-low-birth-weight preterm infants in Brazilian neonatal units. BMC Pediatrics, v. 15, n.113, 2015.

9 GUZMAN CABAÑAS, Juan M.; et al. Factores de riesgo implicados en la necesidad de transfusión sanguínea en recién nacidos de muy bajo peso tratados con eritropoyetina. An Pediatr (Barc), v. 73, n. 6, p. 340-346, 2010. 
10 FREITAS, Brunnella Alcântara C.; FRANCESCHINI, Sylvia do Carmo C. Fatores associados à transfusão de concentrado de hemácias em prematuros de uma unidade de terapia intensiva. Rev Bras Ter Intensiva, v. 24, n. 3, p. 224-229, 2012.

11 DOGRA, Kanchan; et al. Red Cell Transfusion Practices in Neonatal Intensive Care Unit: An Experience from Tertiary Care Centre. Indian J Hematol Blood Transfus, v. 34, n. 4, p. 671-676, 2018.

12 PORTUGAL, Carolina Augusta A.; et al. Transfusion practices in a neonatal intensive care unit in a city in Brazil. Rev bras hematol hemoter, v. 36, n. 4, p. 245-249, 2014.

13 DICKY Odile; et al. Delayed umbilical cord clamping in preterm infants born before 37 weeks of gestation: A prospective observational study. Arch Pediatr, v. 24, n. 2, p. $118-$ $125,2017$.

14 JOSEPHSON, Cassandra D.; MEYER, Erin. Neonatal and pediatric transfusion practice. In: GROSSMAN, Brenda J.; HILLYER, Christopher D.; WESTHOFL, Connie M. (Org). AABB technical manual, Bethesda: ABB Press, 2014. P. 571-588.

15 KIRPALANI, Haaresh; WHYTE, Robin K. What is new about transfusions for preterm infants? An Update. Neonatology, v. 115, n. 4, p. 406-410, 2019.

16 KEIR, Amy.; et al. Adverse effects of red blood cell transfusions in neonates: a systematic review and meta-analysis. Transfusion, v. 56, n. 11, p. 2773-2780, 2016

17 SANTOS, Amélia Miyashiro N.; et al. Variability on red blood cell transfusion practices among Brazilian neonatal intensive care units. Transfusion, v. 50, n. 1, p.150-159, 2010.

18 GHIRARDELLO, Stefano; et al. Effects of Red Blood Cell Transfusions on the Risk of Developing Complications or Death: An Observational Study of a Cohort of Very Low Birth Weight Infants. Am J Perinatol, v. 34, n. 1, p. 88-95, 2017.

19 HOWARTH, Claire; BANERJEE, Jayanta; ALADANGADY, Narendra. Red Blood Cell Transfusion in Preterm Infants: Current Evidence and Controversies. Neonatology, v. 114, n. 1, p. 7-16, 2018.

20 OHLS, Robin K.; et al. A randomized, masked study of weekly erythropoietin dosing in preterm infants. J Pediatr, v. 160, n. 5, p.790-5, 2012.

21 MIMICA, Ana Flávia M.A.; et al. A very strict guideline reduces the number of erythrocyte transfusions in preterm infants. Vox Sang, v. 95, n. 2, p. 106-111, 2008.

22 NAYERI, Fatemeh; et al. Evaluation of a new restricted transfusion protocol in neonates admitted to the NICU. Med J Islam Repub Iran, v. 28, n.119, 2014. 